\title{
Preprocessing Endoscopic Images of Colorectal Polyps
}

\author{
A. Horváth ${ }^{1}$, Sz. Spindler ${ }^{1}$, M. Szalai ${ }^{2}$, I. Rácz ${ }^{2}$ \\ ${ }^{1}$ Széchenyi István University, Department of Physics and Chemistry \\ Győr, Hungary \\ ${ }^{2}$ Petz Aladar County and Teaching Hospital, Györ, Hungary \\ E-mail: horvatha@sze.hu
}

Abstract: Classification of polyps in the human colorectum is a hot topic of gastroenterology. The current advancements in devices of endoscopy made it possible to product high resolution images of polyps with different light filters, e.g. with narrow band imaging (NBI) system. There exists several human classification methods that helps the doctor to decide whether a specific polyp is risky (i.e. it can be turn into cancer) or not. To overcome the limits of human classification skill, a digital image processing method was developed by the authors of this paper.

This paper summarizes the preprocessing method of this software, which is not a trivial task, because during the inspection of a patient, the diagnostician has limited control of light and geometry. Therefore the surface of the polyp is not uniformly illuminated, and prominent flares could appear on the images quite often. These circumstances makes it hard to detect the surface patterns precisely.

In this paper a lot of variants for this preprocessing task is presented. The best of them appears to reproduce the geometrical parameters of the patterns in 1$5 \%$ relative error which is enough for the classification method. The proposed method can be applied to another problems, where the precise measurement of patterns in highly variable illuminating conditions is needed.

Keywords: image processing, thresholding, illumination

\section{Motivation}

Colorectal cancer is one of the most lethal health problem of our age [1]. Many studies pointed out that this disease is responsible for almost the highest cancer related mortality rate in Europe. This is one of the reasons why clinical experts are working together with other field's specialists to find new diagnostic methods for this type of cancers, preferably in early stage. 
Polyps are distortions of the human colorectum that can be detected as 1-20 mm diameter outgrowths the inner surface of the colorectum. Some type of polyps develop into cancer with high probability, meanwhile others remain polyps. In the former case endoscopic poly removal is recommended, in the latter case it is not necessary, if the size is not too big to form an obstacle in the colorectum.

The most accurate method of polyp classification is histology: colonoscopes are usually able to cut off small samples of suspicious part of the tissues (biopsy) and these samples can be examined later. This method has significant drawbacks: histology is time-consuming and requires specialist. Moreover, the biopsy itself generates approximately the same injury as the complete removal of the suspicious polyp. To circumvent these difficulties a lot of methods was developed and are under development to classify polyps based on endoscopic images only. In an ideal case, a good method can help to decide whether the polyp is dangerous (potentially can evolve into cancer) or not. Hence the patient have to bear the negative consequences of polyp ablation the procedure should be carried out only if it is really necessary.

Significant improvement in colonoscope hardware during the last decade made it possible to produce high resolution images and to choose some special light sources or filters. For example, the so called "Narrow Band Imaging" (NBI) filter light source into the 400 to $450 \mathrm{~nm}$ wavelength domain. In this domain the human blood absorbs the light with a very high efficiency, therefore the contrast among vessels and other tissues become more significant comparing to the white light illumination.(See Fig. 1 and [3].)

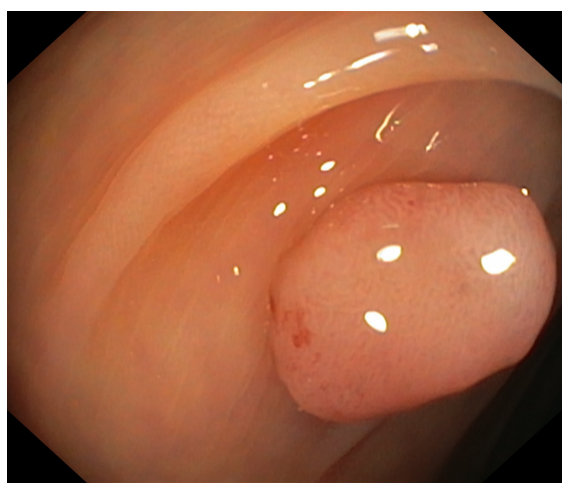

White

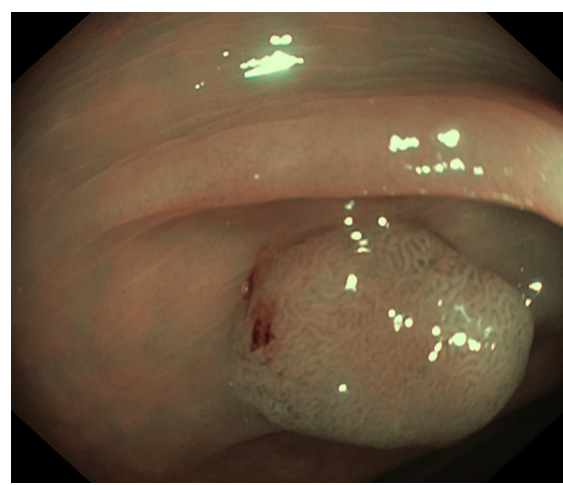

NBI

Figure 1. Same polyp with white and NBI illumination

During the recent years several classification methods were developed for these high resolution and/or NBI images [4]. All of them agreed on using the surface patterns as a key feature but take other parameters into account too. It takes a lot of time and practice to detect and classifiy surface structures like polyps and different kind of lesions. As many 
studies pointed out the learning curve of classification is a very time consuming activity however there are specific trainings exist to facilitate the process. (See [6] and [7].)

The goal of this research is to develop a software that can decide whether a polyp can potentially evolve into cancer or not. In medical terminology doctors refer to the "harmless type" as hyperplastic (HP) and the others commonly called as non-hyperplastic (non-HP). The latter group can be divided into several sub classes, but it is out of our scope at this phase of the research.

\section{Overview of the image processing method}

To overcome the limits of human classification ability, a few research teams have been working on a method based on digital image processing.Széchenyi István University and Petz Aladár County Teaching Hospital also has a joint research in this field. The medical doctors in this project collected approximately 200 cases, with both high resolution NBI images and histological results. They classified these cases with standard Kudo and NICE methods. Authors of this paper developed a digital image processing software which can produce similar statistical accuracy that that of human classification methods. The main steps are:

1. Marking the surface of polyp in the picture. This is the "region of interest" (ROI) of the further investigations.

2. Preprocessing and noise filtering.

3. Identifying the small dark spots and measuring the geometrical parameters.

4. Classify the spots based on geometrical parameters with Quadratic Discriminant Analysis. (QDA)

5. Classify the polyp based on the statistics of spots and some global properties.

The whole method is too complex to publish it in one single paper, therefore only the 2nd and 3rd steps (preprocessing and identifying) will be described here in detailed. also several possible alternatives are going to be examined and the partly new type of adaptive thresholding method is presented.

The other steps are summarized here briefly.

\subsection{ROI selection}

Selection of the Region of Interest (ROI) is not trivial on endoscopic images. As it is clear from Fig. 1, it is not a simple task to a computer to find a polyp itself. Additional difficulty is that parts of the image can be distorted by humours, and prominent flare effects can arise. All of these areas are bad for processing and should not be included in ROI. 
However the flares can be locked out automatically, the other problems would require too sophisticated algorithm. At this stage of the research human interaction is inevitable and for this reason an expert on this field were asked to find these regions and cut the non-ROI parts out from the original picture with an image-editing program (GIMP).

\subsection{Classification}

The method used to assign polyps to one of the two groups (HP and non-HP) is based on normal human classification methods. A key aspect of them is to categorize the glands on the surface of polyps by their shapes. Based on Kudo-Tsuruta classification (see [5]), 4 categories were used: from the small, circular glands were called "sI"-type, to "sIV" category that stands for entirely irregular shape with filamentary structure. "sII" and "sIII" are transitional shapes, the former is for compact, but not circular shapes which indicates slightly distorted, the latter is for highly prolonged but not filamentary structure. Fig. 2 shows examples for each type.

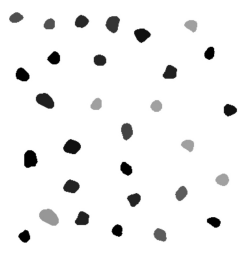

SI

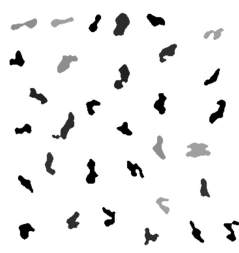

SII

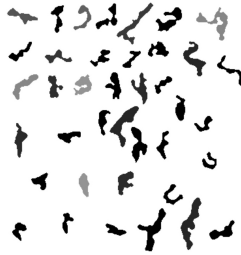

SIII

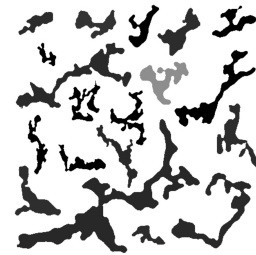

SIV

Figure 2. Examples for individual glands of different types

Based on prior researches sI and sII type shapes are indicators of HP, while sIII and sIV glands imply non-HP polyp. Hence the exposed method classifies the spots on the polyp image, calculates the relative area of sIII and sIV spots (with higher weight for sIV and sI spots). Higher value of this relative area indicates non-HP type.

To find a classification method for glands a lot of scale-invariant geometrical parameters were calculated. It turned out that the following three parameters can characterize these maculas rather accurately:

- Circularity: $C=4 \pi A / P^{2}$, where $A$ is the area $P$ is the perimeter of the spot.

- Solidity: $S=A / A_{h}$, where $A_{h}$ is the area of the convex hull.

- Hu-0: $H$, the 0th Hu-invariant. (See [10])

To determine a spot type i.e to classify it we used QDA. As training set we extracted 1200 maculas from original colonoscopic images labeled by medical doctors who has huge 
experience on this field. The resulted QDA method can rank the shapes into the 4 sets with high precision (higher than $95 \%$ ) based on $C, S$ and $H$ parameters.

An another indicator that correlates with the HP-non-HP groups is the width of the intensity histogram of polyp surface. Based on the previously mentioned "relative area" and this contrast-like parameter a classification method was developed. The statistical accuracy (85-90\%) reached the accuracy of human classification with Nice and Kudo methods. (The reference in accuracy calculation was the result of histology.)

The details of these classification methods was published in [8].The statistical results can be found in [9]. Improved version will be published in a further paper.

\section{The preprocessing}

\subsection{The aim of preprocessing step}

Preprocessing in this classification software starts with noise filtering to reduce the impact of the camera's noise. After trying 3-4 different alternatives, median filter was selected as an appropriate step for doing this.

In endoscopic practice the medical doctor has limited freedom in the geometry and light sources, therefore significant illumination differences are perceptible in the images, even inside the ROI of one polyp. Fig. 3. shows three such examples.
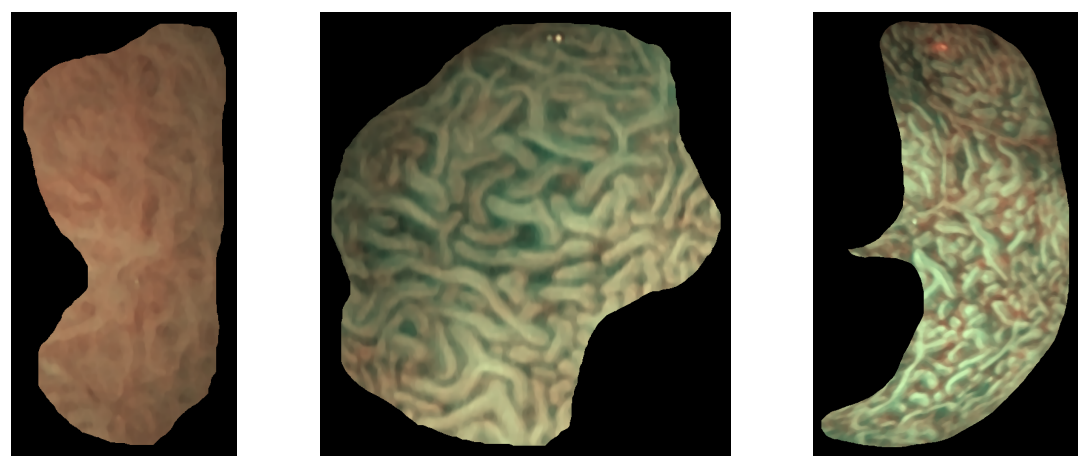

Figure 3. Examples of non-uniform illumination inside a polyp surface.

It is obvious that change of illumination makes it hard to detect the spots and measure their geometrical parameters accurately, and biases the histogram of the image. For this reason the main aim of preprocessing is to transform out the change of illumination.

Since surface of polyps are more or less ellipsoidal shapes, the characteristic size of illumination variation is in the order of the size of ROI. The standard high-pass filtering 
with Fourier transformation is not appropriate in this problem, because the ROI has irregular shape and it causes significant windowing bias.

\subsection{Brightness correction step}

Fig. 3 shows that illumination changes slowly but not monotonous inside the ROI. The basic idea of eliminating this effect is to approximate the illumination change with a "brightness function". Ratio of the image pixel values and this brightness function will describe the surface itself.

In absence of 3D data of polyp surface and light source geometry, a plausible approximation of this brightness function must be calculated from image data. The surface of the polyp has similar average properties across the whole ROI therefore the base assumption is to fit a function that slowly changes to the image intensity. Eventually this will produce a good approximation of the brightness function. After some consideration, third order, two variable polynomials was selected for this purpose.

To formulate the brightness correction step it is practical to use $(x, y)$ coordinates in the image plane so that the image fit into the unit square. If the image has dimensions $N_{x}$ and $N_{y}$, then the pixel in the $i_{x}$-th column and $i_{y}$-th row will have the following coordinates:

$$
x=i_{x} \cdot \delta, \quad y=i_{y} \cdot \delta, \quad \text { where } \quad \delta=1 / \max \left(N_{x}, N_{y}\right) .
$$

The filtered image was converted to grayscale image with standard sRGB weights:

$$
L\left[i_{x}, i_{y}\right]=0.299 R\left[i_{x}, i_{y}\right]+0.587 G\left[i_{x}, i_{y}\right]+0.114 B\left[i_{x}, i_{y}\right],
$$

where $R[], G[]$ and $B[]$ are the color components of the image and $L[]$ is the resulted grayscale image. (All color components are transformed into $[0,1]$ interval.)

Let's define the following 3rd order 2 variable function in the plane of the image:

$$
l(x, y)=A_{00}+A_{10} x+A_{01} y+A_{20} x^{2}+A_{11} x y+A_{02} y^{2}+A_{30} x^{3}+A_{21} x^{2} y+A_{12} x y^{2}+A_{03} y^{3} .
$$

Method of least squares was used to find the $A_{i j}$ parameters in this equation, when $l(x, y)=l\left(i_{x} \cdot \delta, i_{y} \cdot \delta\right)$ approximates $L\left[i_{x}, i_{y}\right]$. It means that the following function was minimized:

$$
W\left(A_{00}, A_{10}, A_{01}, \ldots, A_{03}\right)=\sum_{\left(i_{x}, i_{y}\right) \in R O I}\left(L\left[i_{x}, i_{y}\right]-l\left(i_{x} \cdot \delta, i_{y} \cdot \delta\right)\right)^{2} .
$$

(Note that "[]" means array indexes while "()" is for function arguments. ) 
Since $l(x, y)$ depends linearly on $A_{k l}$ weights, extremal problem (4) can be solved explicitly with the traditional method of least squares. If we use stars for the optimal values $\left(A_{00}^{*}, A_{10}^{*}, \ldots\right)$, the approximation of average brightness will be:

$$
l^{*}(x, y)=A_{00}^{*}+A_{10}^{*} x+A^{*} 01 y+A_{20}^{*} x^{2}+\ldots+A_{03}^{*} y^{3} .
$$

Using this function, the brightness corrected, but not normalized image will be:

$$
L_{b}\left[i_{x}, i_{y}\right]=\frac{L\left[i_{x}, i_{y}\right]}{\max \left(0.2, l^{*}\left(i_{x} \delta, i_{y} \delta\right)\right)} .
$$

Applying the "max" function in the denominator is for safety: in very rare occasions $l^{*}$ has very low, even 0 or less value at some points. In this case it is not good for brightness correction.

The final step in this brightness correction step is the normalization:

$$
L_{b, n}\left[i_{x}, i_{y}\right]=\frac{L_{b}\left[i_{x}, i_{y}\right]}{\max \left(L_{b}\left[i_{x}, i_{y}\right]\right)},
$$

where the "max" is for the pixels of ROI.
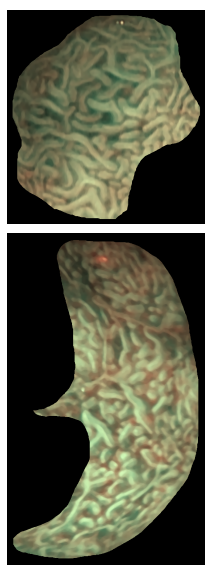

$R, G, B$
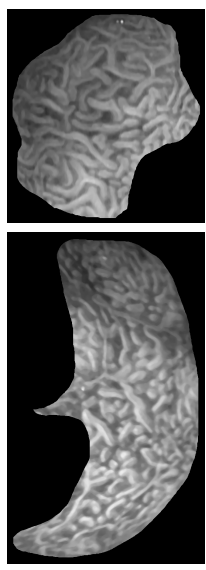

L
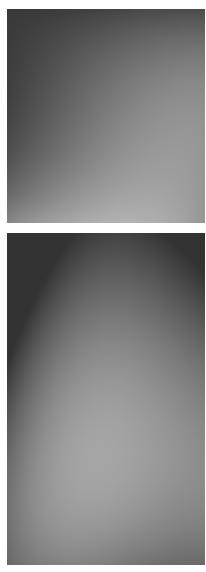

$l^{*}$
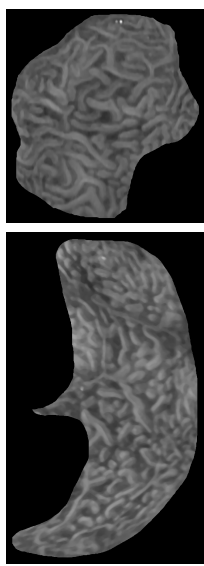

$L_{b, n}$

Figure 4. Steps of brightness correction. See the text for annotations.

Fig. 4 shows two examples: original image, grayscale version, best-fit $l^{*}$ and the final corrected and normalized $L_{b, n}$ image is displayed. It is clear that this type of brightness correction works well on arbitrary ROI, does not produce artifacts and reaches its aim: transforms out the effect of variable illumination. 


\subsection{Thresholding}

On a grayscale image many detail is still hiding although most of these details are surplus. It is necessary to reduce the detailed intensity information into 1 bit: a pixel is inside of a "dark" spot or not. After this step we can measure the geometrical parameters of the spot.

The simplest method of this binarization is to use a fix threshold value for the whole image, i.e. the binary image will be:

$$
B\left[i_{x}, i_{y}\right]=\left\{\begin{array}{ll}
1, & \text { if } L\left[i_{x}, i_{y}\right]>T \\
0, & \text { otherwise }
\end{array} .\right.
$$

Selecting a global $T$ threshold value is not self-explanatory. There are algorithms that can pick up a relatively good one, like the Otsu's method. ([11]) It provides an acceptable result if the average brightness of the picture is uniform, therefore it makes sense to apply it after the brightness correction.

\subsubsection{Adaptive thresholding}

However, choosing an adaptive threshold value is often makes the binarization more accurate. The usual way to do this is to calculate a place-dependent threshold value from a smoothed version of the image:

$$
T\left[i_{x}, i_{y}\right]=\sum_{l=-k}^{k} \sum_{j=-k}^{k} L\left[i_{x}+j, i_{y}+l\right] \cdot K[j, l],
$$

where $K$ is the kernel of the smoothing operator, $k$ is the "radius" of kernel. $K$ is assumed to be normalized: $\sum_{l=-k}^{k} \sum_{j=-k}^{k} K[l, j]=1$.

(9) means a simple convolution between $L$ and $K$, therefore it can be rewritten as:

$$
T=L * K
$$

where '*' is the sign of convolution.

The usual choices for $K$ are: uniform and Gaussian.

- Uniform kernel: $K[j, l]=1 /(2 k+1)^{2}$ for all $j$ and $l$.

- Gaussian kernel: $K[j, l]=n \cdot e^{-\left(j^{2}+l^{2}\right) /\left(2 \sigma^{2}\right)}$ where $\sigma$ is the deviation and $n$ is a suitable normalization factor.

With that $T[]$ threshold array the binary image will be the following:

$$
B\left[i_{x}, i_{y}\right]= \begin{cases}1, & \text { if } L\left[i_{x}, i_{y}\right]>T\left[i_{x}, i_{y}\right]+C \\ 0, & \text { otherwise }\end{cases}
$$


The offset value $C$ is for fine-tuning: setting it to positive or negative value makes less or more 1-s in binary image.

The (9) formula is a convolution and can be calculated very efficiently with Fast Fourier Transformation for large image and kernel sizes. Therefore if we know the values of $L[]$, the kernel $K$ and $C$ offset value, binary image $B$ can be determined in short time. This method is often referred as "adaptive thresholding".

\subsubsection{Conditional adaptive thresholding}

The original adaptive thresholding method has obvious problems if $L[]$ is not known for all the domain, and this is the case in endoscopic images of polyps, because of the irregular shape of ROI. Outside the ROI the pixels give no information about the polyp itself, therefore non-ROI pixels should be excluded from calculations. In other words: values of $L[]$ is unknown outside of the ROI.

A simple solution could be to use a constant value (e.g. 0) outside the ROI. However it is obvious that this would highly distort the result of convolution near the borders. A more sophisticated method is to use some "inpainting" method to fill the non-ROI pixels based on near-border values. This method was tested and rejected because a large area around the ROI should be filled with approximated values and the tests showed very unnatural intensity distributions. An additional drawback of inpainting is the high CPU-usage.

Instead of filling with constant or inpainted values an another approach was used. (9) can be interpreted as a weighted average of $L[]$ values (plus an offset). The sum of weights in $K$ kernel is 1 in this case. It is easy to modify this formula to calculate the weighted average only for ROI pixels:

$$
\begin{aligned}
T\left[i_{x}, i_{y}\right] & =\frac{1}{D\left[i_{x}, i_{y}\right]} \sum_{(j, l) \mid\left(i_{x}+j, i_{y}+k\right) \in R O I} L\left[i_{x}+j, i_{y}+l\right] \cdot K[j, l] \\
D\left[i_{x}, i_{y}\right] & =\sum_{(j, l) \mid\left(i_{x}+j, i_{y}+k\right) \in R O I} K[j, l] .
\end{aligned}
$$

The long formula below the sum sign means that the summation is for those $(j, l)$ pairs, when $\left(i_{x}+j, i_{y}+l\right)$ refers to a point inside the ROI.

It is obvious that (12) gives the same result as (9) for pixels deeply inside the ROI and always gives a normalized, weighted average of ROI pixels that are at most $k$ distance in infinity norm. This kind of smoothing can work for arbitrary shaped ROIs, but in this form it is very expensive in computational steps. Fortunately it can be accelerated by reduction 
into two separate convolution. Let's define the characteristic function of the ROI:

$$
Q\left[i_{x}, i_{y}\right]= \begin{cases}1, & \text { if }\left(i_{x}, i_{y}\right) \text { is inside ROI } \\ 0, & \text { otherwise }\end{cases}
$$

Using this function (12) can be written as:

$$
\begin{aligned}
T\left[i_{x}, i_{y}\right] & =\frac{1}{D\left[i_{x}, i_{y}\right]} \sum_{l=-k}^{k} \sum_{j=-k}^{k}\left(L\left[i_{x}+j, i_{y}+l\right] Q\left[i_{x}+j, i_{y}+l\right]\right) K[j, l] \\
D\left[i_{x}, i_{y}\right] & =\sum_{l=-k}^{k} \sum_{j=-k}^{k} Q\left[i_{x}+j, i_{y}+l\right] K[j, l] .
\end{aligned}
$$

On the right sides we have two simple convolutions:

$$
\begin{aligned}
T & =\frac{1}{D}(L \cdot Q) * K \\
D & =Q * K .
\end{aligned}
$$

Both convolution can be effectively evaluated with FFT based algorithm. The pixelwise division with $D$ array elements is a cheap operation but can occur division by zero operations. However, it is easy to show that it will never happen for points of ROI for all plausible $K$ kernels. To be more precise: if all elements of $K$ are nonnegative and $K[0,0]>0$, then $D\left[i_{x}, i_{y}\right]>Q\left[i_{x}, i_{y}\right] K[0,0]=K[0,0]>0$ for all $\left(i_{x}, i_{y}\right) \in$ ROI.

For non-ROI pixels, where $D$ can be $0, T$ is arbitrary and the division with pixel of $D$ is unnecessary, because values of $T$ is used only in (11) to get the binarized image inside the ROI.

The steps in (15) is analogue with the conditional probability or conditional expected value calculations based on Bayes' theorem. The smoothing operator can be interpreted as a calculation method for a expected value of a pixel: the expected value depends on the values of neighbours through a weighted average calculation, where the close neighbours has higher weight.

\section{Testing method}

\subsection{Test problems}

The methods described above can be combined in many ways. For example, one can use Otsu's method for thresholding with or without brightness correction, or use adaptive thresholding with uniform or Gaussian kernel, or the last method: conditional adaptive 
thresholding, etc. To choose the best method, a set of test problems was constructed which are similar to the real polyp images but the list of dark spots and exact value of their geometrical parameters are known.

1. Four "flat" image were created using the categorized examples of dark spots. These were the same as the four image on Fig. 2. Each flat image contains spots of the same type which is useful to identify type-specific problems and inaccuracies.

2. Three different illumination distribution was created: a circular, an ellipsoidal and an ellipsoidal with flares. (See Fig. 5.)

3. The 12 test images were constructed by multiplication of flat images and illumination distributions with added Poisson-noise.

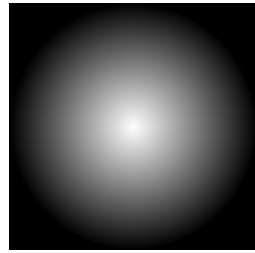

ID1

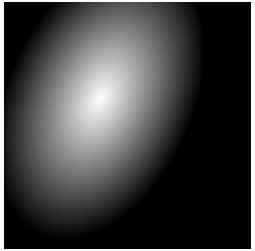

ID2

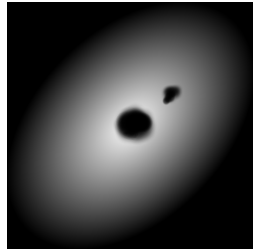

ID3

Figure 5. Illumination distributions of test problems.

The resulted 12 test images (combination of 4 flat and 3 illumination distribution images) has similar noise and brightness properties to real polyp images, but the exact geometrical parameters can be measured on the flat images. Two of them is displayed on Fig. 6. As a short notation "T- $x / y$ " will refer to the test problem which was derived from $x$-th flat image and $y$-th illumination distribution.

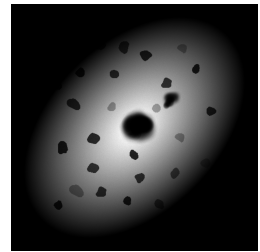

T-1/3: sI*ID3+ noise

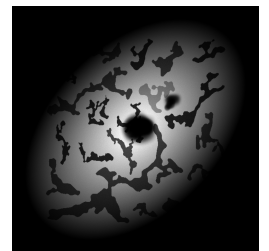

T-4/3: sIV*ID3 + noise

Figure 6. Two of the 12 test images.

Note that the test images does not imitate the interesting parts of polyp images in every aspect. For example, on real images spots of all category can be found in one image, the spots are more randomly placed and sometimes are very close to each other. These 
simplified test images make it possible to measure the accuracy of preprocessing for every spot category and clear separation of spots prevent from unwanted artifacts.

On the illumination distribution images the pixel values are 0 outside the simulated polyps and inside the simulated flares, otherwise they are positive. Therefore the ROI of a test image is the area, where the illumination distribution pixels has positive values.

\subsection{Testing method}

The basic idea for testing is to measure the "exact" geometrical parameters on the original flat images (Fig. 2) and compare them to the preprocessed image values. However, some care is needed to take, because if a spot on a flat image is fully or partly outside of the ROI (nonzero pixels on Fig. 5) of a test image, one can not expect to find it after preprocessing. Therefore to get reference values of geometrical parameters, the flat images were masked by the ROI of test image and only these inner spots were used as reference.

The measured and stored parameters were the following: map of spots (the pixels for every individual spots), circularity $(C)$, solidity $(S)$, Hu 0 coefficient $(H)$, area $(A)$, perimeter $(P)$.

The 12 test images were preprocessed for a number of combination of preprocessing steps and in each case the same parameters were measured on them.

Using the map of spots on the masked flat and the preprocessed test image, the matching spot-pairs was searched for at first. Two spots was called "matching" if they had at least one common pixel. For every spots on masked flat image the number of matching spots $(M)$ on preprocessed image was calculated. In ideal case $M=1$ for every spot, but if the preprocessing is inaccurate, we can get $M=0$ (no matching spot) or $M>1$ (multiple matching spots). The "miss ratio" of the spot-matching was the ratio of $M \neq 1$ cases and the number of spots. This "miss ratio" characterizes the accuracy of spot-preserving of a particular preprocessing method.

For spots with $M=1$ matching pair, the geometrical parameters were measured also on preprocessed test image and the relative difference between corresponding values was calculated. The average of these relative errors was used to characterize the accuracy of geometrical parameter preservation.

\subsection{The examined preprocessing methods}

In section 3 a lot of possible preprocessing step was described. Some of them has parameters, and some of them are optional. This means that a lot of preprocessing chain can be formed using them. For a summary, see Table 1.

The number of possible preprocessing methods is very high, so all of the test results can 


\begin{tabular}{|l|l|c|c|}
\hline Preproc. step & Variation & Parameters & Abbrev. \\
\hline Median filter & - & radius $(R)$ & - \\
\hline Brightness corr. & - & - & BC \\
\hline \multirow{4}{*}{ Thresholding } & Otsu & - & OT \\
& Adaptive / Uniform kernel & kernel radius ( $k)$ & AThu \\
& Adaptive / Gaussian kernel & kernel radius $(k)$ & AThg \\
& Conditional Adaptive / Gaussian & kernel radius $(k)$ & CATh \\
\hline
\end{tabular}

Table 1. Preprocessing steps used in testing.

not be presented in this paper. The calculations showed that the best parameters are the following:

- Median filter radius: $R=1$ ( $3 \times 3$ size structuring element).

$R=0$ means no median filtering. In this case the noise is not suppressed what leads to big errors. $R>1$ means strong noise reduction but it smooths the boundary of spots and results in high error level in geometrical parameters.

- Adaptive threshold kernel radius: $k=W / 3$, where $W$ is an effective width of the ROI calculated as the ratio of area and diameter of ROI.

A lot of test calculations showed that the kernel radius must be in the magnitude of average width of ROI. Calculations was performed with different values (i.e. $k=W$, $W / 2, W / 3, W / 5 \ldots)$ and the $k=W / 3$ choice appeared to be the best.

\subsection{Implementation}

The described methods were implemented in Python and NumPy module was used for numerical calculations (Brightness correction, CATh). For basic image manipulation, input-output, convolution, standard adaptive thresholding (AThu, AThg) OpenCV was applied. ([12])

\section{Results}

The relative errors of geometrical parameters are denoted with $\mathcal{R}(C), \mathcal{R}(S), \ldots$, the average of them is $\overline{\mathcal{R}}$. The number of spots on masked flat image is $N$, and $N_{1}, N_{0}, N_{m}$ stands for number of unique match, missing spot and multiple matches respectively. The miss ratio is $\mathcal{M}=\left(N_{0}+N_{m}\right) / N$.

A specific preprocessing method is characterized by this average relative error $(\overline{\mathcal{R}})$ and miss ratio $(\mathcal{M})$. In ideal case both one would be 0 . 


\subsection{A detailed calculation result}

Tab. 2 shows the results for T-1/2, T-2/3, T-3/3, T-4/3 test results (the cases for the most difficult ID3 illumination distribution), and for $M=1$ (i.e. for $3 \times 3$ median filter). All of the measured parameters (relative errors of all 5 geometrical parameters and all type of matching errors) is displayed in details. The results are sorted in increasing order of $\mathcal{M}$ inside a test problem and increasing $\overline{\mathcal{R}}$ value if the miss rate is the same.

\begin{tabular}{|c|c|c|c|c|c|c|c|c|c|c|c|c|}
\hline \multicolumn{2}{|c|}{ Method } & \multicolumn{6}{|c|}{ Geometrical Errors } & \multicolumn{5}{|c|}{ Matching Errors } \\
\hline $\mathrm{BC}$ & Thresh & $\mathcal{R}(C)$ & $\mathcal{R}(S)$ & $\mathcal{R}(H)$ & $\mathcal{R}(A)$ & $\mathcal{R}(P)$ & $\overline{\mathcal{R}}$ & $N$ & $N_{1}$ & $N_{m}$ & $\overline{N_{0}}$ & $\mathcal{M}$ \\
\hline \multicolumn{13}{|c|}{$\mathrm{T}-1 / 3$} \\
\hline+ & CATh & 0.072 & 0.017 & 0.003 & 0.024 & 0.043 & $3.18 \%$ & 20 & 20 & 0 & 0 & $0.00 \%$ \\
\hline+ & AThg & 0.082 & 0.025 & 0.003 & 0.014 & 0.044 & $3.36 \%$ & 20 & 20 & 0 & 0 & $0.00 \%$ \\
\hline - & CATh & 0.075 & 0.019 & 0.003 & 0.026 & 0.046 & $3.40 \%$ & 20 & 20 & 0 & 0 & $0.00 \%$ \\
\hline- & AThg & 0.086 & 0.028 & 0.003 & 0.016 & 0.047 & $3.59 \%$ & 20 & 20 & 0 & 0 & $0.00 \%$ \\
\hline+ & AThu & 0.108 & 0.038 & 0.015 & 0.058 & 0.060 & $5.59 \%$ & 20 & 20 & 0 & 0 & $0.00 \%$ \\
\hline - & AThu & 0.170 & 0.112 & 0.212 & 0.401 & 0.717 & $32.25 \%$ & 20 & 19 & 0 & 1 & $5.00 \%$ \\
\hline- & OT & 0.212 & 0.187 & 0.047 & 6.941 & 5.389 & $255.51 \%$ & 20 & 17 & 0 & 3 & $15.00 \%$ \\
\hline+ & OT & 0.046 & 0.012 & 0.022 & 0.162 & 0.110 & $7.05 \%$ & 20 & 15 & 0 & 5 & $25.00 \%$ \\
\hline \multicolumn{13}{|c|}{$\mathrm{T}-2 / 3$} \\
\hline+ & CATh & 0.077 & 0.021 & 0.014 & 0.028 & 0.047 & $3.75 \%$ & 24 & 24 & 0 & 0 & $0.00 \%$ \\
\hline - & CATh & 0.075 & 0.019 & 0.016 & 0.031 & 0.049 & $3.82 \%$ & 24 & 24 & 0 & 0 & $0.00 \%$ \\
\hline - & AThg & 0.100 & 0.041 & 0.010 & 0.020 & 0.055 & $4.53 \%$ & 24 & 23 & 1 & 0 & $4.17 \%$ \\
\hline+ & AThg & 0.096 & 0.038 & 0.011 & 0.026 & 0.057 & $4.56 \%$ & 24 & 23 & 1 & 0 & $4.17 \%$ \\
\hline+ & AThu & 0.160 & 0.071 & 0.019 & 0.088 & 0.096 & $8.69 \%$ & 24 & 22 & 2 & 0 & $8.33 \%$ \\
\hline - & AThu & 0.195 & 0.132 & 0.193 & 1.329 & 0.988 & $56.75 \%$ & 24 & 22 & 2 & 0 & $8.33 \%$ \\
\hline - & OT & 0.262 & 0.242 & 0.278 & 4.959 & 2.765 & $170.12 \%$ & 24 & 20 & 0 & 4 & $16.67 \%$ \\
\hline+ & OT & 0.114 & 0.087 & 0.074 & 0.224 & 0.150 & $12.97 \%$ & 24 & 16 & 0 & 8 & $33.33 \%$ \\
\hline \multicolumn{13}{|c|}{$\mathrm{T}-3 / 3$} \\
\hline+ & CATh & 0.087 & 0.064 & 0.010 & 0.041 & 0.057 & $5.17 \%$ & 29 & 28 & 1 & 0 & $3.45 \%$ \\
\hline - & CATh & 0.083 & 0.065 & 0.010 & 0.046 & 0.058 & $5.23 \%$ & 29 & 28 & 1 & 0 & $3.45 \%$ \\
\hline - & AThg & 0.126 & 0.049 & 0.021 & 0.049 & 0.078 & $6.44 \%$ & 29 & 28 & 1 & 0 & $3.45 \%$ \\
\hline+ & AThg & 0.176 & 0.118 & 0.033 & 0.074 & 0.103 & $10.09 \%$ & 29 & 28 & 1 & 0 & $3.45 \%$ \\
\hline - & AThu & 0.210 & 0.148 & 0.149 & 0.626 & 0.631 & $35.27 \%$ & 29 & 27 & 2 & 0 & $6.90 \%$ \\
\hline+ & AThu & 0.155 & 0.097 & 0.025 & 0.086 & 0.103 & $9.31 \%$ & 29 & 26 & 2 & 1 & $10.34 \%$ \\
\hline - & OT & 0.342 & 0.363 & 0.069 & 12.266 & 6.282 & $386.43 \%$ & 29 & 22 & 0 & 7 & $24.14 \%$ \\
\hline+ & OT & 0.080 & 0.094 & 0.058 & 0.216 & 0.140 & $11.76 \%$ & 29 & 19 & 1 & 9 & $34.48 \%$ \\
\hline \multicolumn{13}{|c|}{$\mathrm{T}-4 / 3$} \\
\hline+ & CATh & 0.070 & 0.057 & 0.012 & 0.034 & 0.047 & $4.40 \%$ & 19 & 19 & 0 & 0 & $0.00 \%$ \\
\hline - & CATh & 0.067 & 0.058 & 0.012 & 0.037 & 0.047 & $4.43 \%$ & 19 & 19 & 0 & 0 & $0.00 \%$ \\
\hline+ & AThg & 0.461 & 0.220 & 0.057 & 0.120 & 0.136 & $19.87 \%$ & 19 & 16 & 3 & 0 & $15.79 \%$ \\
\hline+ & AThu & 0.450 & 0.224 & 0.056 & 0.132 & 0.139 & $20.01 \%$ & 19 & 16 & 3 & 0 & $15.79 \%$ \\
\hline - & AThg & 0.489 & 0.229 & 0.058 & 0.125 & 0.142 & $20.88 \%$ & 19 & 15 & 4 & 0 & $21.05 \%$ \\
\hline - & AThu & 0.355 & 0.162 & 0.033 & 0.127 & 0.119 & $15.92 \%$ & 19 & 14 & 3 & 2 & $26.32 \%$ \\
\hline - & OT & 0.443 & 0.378 & 0.143 & 8.671 & 3.480 & $262.31 \%$ & 19 & 13 & 2 & 4 & $31.58 \%$ \\
\hline+ & OT & 0.263 & 0.151 & 0.115 & 0.232 & 0.187 & $18.94 \%$ & 19 & 11 & 2 & 6 & $42.11 \%$ \\
\hline
\end{tabular}

Table 2. Detailed test results with $R=1$ median filtering for the ID3 illumination distribution for all the flat images.

The most important results can be summarized as follows:

- The Otsu method is just not good enough even with brightness correction. It is in accordance with our preliminary suspicion. Although it should be noted that with 
brightness correction the geometrical error is quite low, but the miss rate is still unacceptably high.

- The classical adaptive thresholding works significantly better with Gaussian kernel compared to uniform one. It is plausible: the uniform kernel uses the same weight factor for far and near pixels.

- The clear winner is the CATh (Conditional Adaptive Thresholding) method with BC (Brightness correction). CATh was surprisingly accurate even without BC step.

- CATh works well in the following manner too: flat images with higher index contain more complex and elongated shapes. The miss rate and average geometrical error remains almost the same with this method for T-4/3 test case (sIV spots) than that of with $\mathrm{T}-1 / 3$ case (sI spots).

The detailed error quantities $\left(\mathcal{R}(C), \mathcal{R}(S), \ldots, N_{m}, N_{0}\right)$ show no interesting results: they are in good correlation with the total parameters $(\mathcal{R}$ and $\mathcal{M})$. Therefore in the following test results only $\mathcal{R}$ and $\mathcal{M}$ will be displayed.

To keep this paper compact, results for ID1 and ID2 illumination distribution is not presented. As they are less complex than the ID3 case, both $\mathcal{R}$ and $\mathcal{M}$ has smaller values than in ID3 cases, but the relative accuracy of preprocessing methods is the same as above.

\subsection{The effect of the median filter's radius}

The ideal radius of median filter $(R)$ was also examined. Higher values mean more noise reduction but can significantly change the shape of the spots. In this paper only the results for the best preprocessing method (CATh with BC) is presented for ID3 illumination distribution: Table 3 shows the miss rates, Table 4 the average geometrical errors.

\begin{tabular}{|c||c|c|c|c|}
\hline & $R=1$ & $R=2$ & $R=3$ & $R=7$ \\
\hline \hline $\mathrm{T}-1 / 3$ & $0.00 \%$ & $0.00 \%$ & $0.00 \%$ & $0.00 \%$ \\
$\mathrm{~T}-2 / 3$ & $0.00 \%$ & $0.00 \%$ & $0.00 \%$ & $0.00 \%$ \\
$\mathrm{~T}-3 / 3$ & $3.45 \%$ & $3.45 \%$ & $0.00 \%$ & $3.45 \%$ \\
$\mathrm{~T}-4 / 3$ & $5.26 \%$ & $0.00 \%$ & $0.00 \%$ & $0.00 \%$ \\
\hline
\end{tabular}

Table 3. Missing rates $(\mathcal{M})$ for the best preprocessing method (CATh with $B C)$ with different radius of median filter $(R)$.

It is clear that $R=1$, which is equivalent with no median filtering is the worst in miss rates. The matching errors arise in the sIII and sIV cases, i.e. when the shapes are more complex. The reason is that in this cases the noise can lead to division of one spot into smaller ones. 


\begin{tabular}{|c||c|c|c|c|}
\hline & $R=1$ & $R=2$ & $R=3$ & $R=7$ \\
\hline \hline $\mathrm{T}-1 / 3$ & $1.83 \%$ & $3.18 \%$ & $3.94 \%$ & $5.71 \%$ \\
$\mathrm{~T}-2 / 3$ & $2.55 \%$ & $3.75 \%$ & $4.94 \%$ & $10.78 \%$ \\
$\mathrm{~T}-3 / 3$ & $4.14 \%$ & $5.17 \%$ & $6.15 \%$ & $13.78 \%$ \\
$\mathrm{~T}-4 / 3$ & $3.90 \%$ & $4.40 \%$ & $3.80 \%$ & $10.40 \%$ \\
\hline
\end{tabular}

Table 4. Average geometrical errors $(\overline{\mathcal{R}})$ for the best preprocessing method (CATh with $B C)$ with different radius of median filter $(R)$.

With increasing median filter radius the average geometrical error is increases in most of the cases. (There is only one exception: T-4/3 is better with $R=3$ than with $R=2$.)

One can conclude that median filtering is necessary and the optimal value of its radius is 2 or 3 . This is the reason why we displayed $R=2$ cases above.

Note that similar calculations was performed with other noise filtering than median filter, but they could not produce better results and the most of them required parameter-tuning.

\subsection{Test calculation without noise}

As it was shown above, presence of noise makes filtering necessary, but the filtering has negative effect on geometrical parameters. To measure the accuracy of preprocessing methods without the bias of noise, a new text calculation was performed with test images without simulated noise. Naturally, the median filtering was abandoned during the preprocessing method for these images. The results of this noiseless calculations is displayed in Table 5. (For comparison, results with noise and $R=2$ median filtering is printed there.)

\begin{tabular}{|c||c|c|}
\hline & with noise $(R=2)$ & without noise \\
\hline \hline $\mathrm{T}-1 / 3$ & $3.18 \%$ & $1.65 \%$ \\
$\mathrm{~T}-2 / 3$ & $3.75 \%$ & $2.54 \%$ \\
$\mathrm{~T}-3 / 3$ & $5.17 \%$ & $4.87 \%$ \\
$\mathrm{~T}-4 / 3$ & $4.40 \%$ & $3.73 \%$ \\
\hline
\end{tabular}

Table 5. Average geometrical errors $(\overline{\mathcal{R}})$ for the $C A T h+B C$ method: calculation with and without simulated noise.

These average relative error values show the accuracy of the BC+CATh method itself, while the difference between two columns shows the effect of the noise. We can conclude that the noise increased the geometrical errors only with approximately $1 \%$ and the own error of $\mathrm{BC}+\mathrm{CATh}$ method is in the order of $2-5 \%$, which is adequately low for our purposes. 


\section{Conclusion}

The results displayed above show that combination of brightness correction (BC) and conditional adaptive thresholding (CATh) steps is an appropriate method for preprocessing colonoscopic images of polyps. This method preserves the spots and their geometrical parameters more accurately than classical adaptive thresholding ones. The described methods also provide a firm base for further research in the field of automatic polyp classification, but CATh seems to be useful in other areas, where smoothing or adaptive thresholding calculations are needed on an irregular region of interest.

\section{References}

[1] Neumann H, Vieth M, Fry LC, Günther C, Atreya R, Neurath MF, Mönkemüller $\mathrm{K}$ : Learning curve of virtual chromoendoscopy for the prediction of hyperplastic and adenomatous colorectal lesions: a prospective 2-center study; Gastrointestinal Endoscopy, Vol. 78, No. 1, pp 444-449, 2013

DOI:10.1016/j.gie.2013.02.001

[2] Rex DK: Colonoscopy. Gastrointestinal Endoscopy, Vol. 78, No. 3, 2013

DOI:10.1016/j.gie.2013.06.025

[3] Fiori M, Musé P, Sapiro G: A Complete System for Candidate Polyps Detection in Virtual Colonoscopy International Journal of Pattern Recognition and Artificial Intelligence Vol. 28, No. 7, 2014

DOI: $10.1142 / \mathrm{S} 0218001414600143$

[4] Tomonori Yano, Hironori Yamamoto, Keijiro Sunada, Tomohiko Miyata, Michiko Iwamoto, Yoshikazu Hayashi, Masayuki Arashiro, Kentaro Sugano: Endoscopic classification of vascular lesions of the small intensitine; Gastrointestinal Endoscopy, Vol. 67, No. 1, pp. 196-172, 2008

DOI:10.1016/j.gie.2007.08.005

[5] Yoshito Takemura, Shigeto Yoshida, Shinji Tanaka, Keiichi Onji, Shiro Oka, Toru Tamaki, Kazufumi Kaneda, Masaharu Yoshihara, Kazuaki Chayama: Quantitative analysis and development of a computer-aided system for identification of regular pit patterns of colorectal lesions; Gastrointestinal Endoscopy, Vol. 72, No. 5, pp.10471051,2010

DOI:10.1016/j.gie.2010.07.037

[6] Arber N, Spicak J, Racz I, Zavoral M, Breazna A, Gerletti P, Lechuga MJ, Collins N, Rosenstein RB, Eagle CJ, Levin B: Five-year analysis of the prevention of colorectal sporadic adenomatous polyps trial, American Journal Of Gastroenterology Vol. 106, No. 6, pp. 1135-1146. : 2011

DOI:10.1038/ajg.2011.116 
[7] Durcsán H, Szabó A, Kovács V, Szalai M, Kiss Gy, Lakó K, Gyulyás J, Regőczi H, Rácz I: Population based Colorectal Cancer Screening; preliminary results from Győr, Zeitschrift Für Gastroenterologie 5, pp. 53, 2015

[8] Horváth A, Spindler Sz, Rácz I, Szalai M: Kolonoszkópiás polipképek számítógépes kategorizálása; In proceedings of: "Képfeldolgozók és Alakfelismerók Társaságának 10. országos konferenciája.” Kecskemét, Hungary, 2015.01.27-30., pp. 505-514, 2015

[9] Rácz I, Horváth A, Szalai M, Spindler S, Kiss G, Regöczi H, Horváth Z: Digital Image Processing Software for Predicting the Histology of Small Colorectal Polyps by Using Narrow-Band Imaging Magnifying Colonoscopy. Gastrointestinal Endoscopy, Vol. 81, No. 5, AB259, 2015

[10] $\mathrm{Hu}$, Ming-Kuei: Visual pattern recognition by moment invariants. Information Theory, IRE Transactions on 8.2, pp. 179-187: 1962 DOI 10.1109/TIT.1962.1057692

[11] Otsu, Nobuyuki: A threshold selection method from gray-level histograms. Automatica 11 , no. 285-296 pp. 23-27, 1975

[12] Bradski G, Adrian K: Learning OpenCV: Computer vision with the OpenCV library. O’Reilly Media, Inc., 2008 